\title{
Variance-dependent neural activity in an unvoluntary averaging task
}

\author{
Rémy Allard $^{1,2}$ (1) $\cdot$ Stephen Ramanoël ${ }^{1} \cdot$ Daphné Silvestre $^{1} \cdot$ Angelo Arleo $^{1}$
}

Accepted: 1 December 2020 / Published online: 27 January 2021

(C) The Psychonomic Society, Inc. 2021

\begin{abstract}
Ensemble statistics of a visual scene can be estimated to provide a gist of the scene without detailed analysis of all individual items. The simplest and most widely studied ensemble statistic is mean estimation, which requires averaging an ensemble of elements. Averaging is useful to estimate the mean of an ensemble and discard the variance. The source of variance can be external (i.e., variance across the physical elements) or internal (i.e., imprecisions in the estimates of the elements by the visual system). The equivalent noise paradigm is often used to measure the impact of the internal variance (i.e., the equivalent input noise). This paradigm relies on the assumption that the averaging process is equally effective independently of the main source of variance, internal or external, so any difference between the processing when the main source of variance is internal or external must be assumed not to affect the averaging efficiency. The current fMRI study compared the neural activity when the main variance is caused by the stimulus (i.e., high variance) and when it is caused by imprecisions in the estimates of the elements by the visual system (i.e., low variance). The results showed that the right superior frontal and left middle frontal gyri can be significantly more activated when the variance in the orientation of the Gabors was high than when it was low. Consequently, the use of the equivalent noise paradigm requires the assumption that such additional neural activity in high variance does not affect the averaging efficiency.
\end{abstract}

Keywords Ensemble statistics $\cdot$ Averaging $\cdot$ Orientation $\cdot$ Noise $\cdot$ fMRI

\section{Introduction}

We have the subjective impression that we can perceive the entire visual scene at a glance, but the visual system has limited resources, and the entire visual scene cannot be simultaneously processed with optimal efficiency. For instance, some processing requires the focus of attention to be optimal, but attention can only be divided among few items (Pylyshyn \& Storm, 1988; Treisman \& Gelade, 1980). Nevertheless, the visual system does not completely discard the unattended information of the visual scene, as some global information can be perceived even in the absence of focal attention (for a review, see Whitney \& Yamanashi Leib, 2018). Ensemble statistics of a visual scene can be estimated to provide a gist

Rémy Allard and Stephen Ramanoël contributed equally to this work.

Rémy Allard

remy.allard@umontreal.ca; http://www.aging-vision-action.fr

1 Sorbonne Universités, INSERM, CNRS, Institut de la Vision, 17 rue Moreau, F-75012 Paris, France

2 School of optometry, Université de Montréal, 3744 rue Jean-Brillant, local 260-7, Montreal, Quebec H3T 1P1, Canada of the visual scene without detailed analysis of all individual items. The process of extracting ensemble statistics is often described as "obligatory" or "compulsory" (Fischer \& Whitney, 2011; Parkes, Lund, Angelucci, Solomon, \& Morgan, 2001) as attention may not necessarily be required (Alvarez \& Oliva, 2008, 2009; Bronfman, Brezis, Jacobson, \& Usher, 2014; Chong \& Treisman, 2005).

The simplest and most widely studied ensemble statistic is mean estimation, which requires averaging an ensemble of elements. Averaging is useful to estimate the mean of an ensemble and discard the variance. When the variance across elements is high, the ability to estimate the mean of the a priori distribution (e.g., orientation discrimination threshold) depends on the averaging efficiency (also referred to as calculation efficiency or sampling efficiency), which quantifies the observer's performance relative to the ideal performance (e.g., Beaudot \& Mullen, 2006). Since the correctness of the answer is defined relative to the mean of the a priori distribution, the ideal performance is limited by the variance of the a priori distribution.

When the variance is low (e.g., all identical elements), the performance of a human observer cannot solely depend on the averaging efficiency, as performance is also limited by imprecisions in the estimates of the elements (i.e., internal noise) by the visual system. Consequently, in absence of external 
variance, the ability to estimate the mean of the a priori distribution is typically presumed to depend on two factors (Beaudot \& Mullen, 2006; Dakin, 2001, 2015; Dakin, Bex, Cass, \& Watt, 2009; Dakin, Mareschal, \& Bex, 2005; Mansouri, Allen, Hess, Dakin, \& Ehrt, 2004; Mareschal, Bex, \& Dakin, 2008; Tibber et al., 2015): the variance introduced by the visual system (typically referred to as internal noise) and the averaging efficiency of the effective stimulus (i.e., the stimulus + orientation-jitter due to imprecisions in samples estimates). The equivalent noise paradigm is often used to evaluate the impact of these imprecisions. Knowing the performance in absence of noise and the effective averaging efficiency in absence of noise enables one to derive the internal variance due to imprecisions in samples estimates. However, the effective averaging efficiency in absence of noise cannot be directly measured. Nevertheless, by assuming (usually implicitly) that the effective averaging efficiency in absence of noise is the same as the averaging efficiency measured in high noise (i.e., the noise-invariant processing assumption; Allard \& Cavanagh, 2011; or the contrastinvariant calculation assumption; Pelli, 1990), it is possible to derive the internal variance based on the performance in absence of noise and in high noise. Consequently, this assumption is critical for evaluating the internal noise using the equivalent noise paradigm.

The equivalent noise paradigm is more commonly used for contrast thresholds and white pixel noise, and relies on the noise-invariant processing assumption (Allard \& Cavanagh, 2011, 2012; or the contrast-invaraint processing assumption; Pelli, 1990): The calculation efficiency measured in high noise is assumed to be the same as the effective calculation efficiency in low noise. The presence of high white pixel noise obviously increases neural activity, so to use the equivalent noise paradigm, one must assume that this additional neural activity in high noise does not affect the calculation efficiency. This assumption has been criticized by Baker and colleagues (Baker \& Meese, 2012; Baker \& Vilidaite, 2014; Baldwin, Baker, \& Hess, 2016), who argue that white noise may compromise the use of the equivalent noise paradigm because it introduces neural activity that can interfere with the processing of the target (but see Allard \& Faubert, 2013, 2014). Although it is obvious that some neural activity is greater in the presence of white pixel noise than the absence of noise due to the greater contrast, it is not obvious whether increasing the variance across elements would increase neural activity. For instance, we would not necessarily expect additional neural activity when adding an orientation jitter to each element of an ensemble.

An important distinction that is not always explicitly stated is that the averaging process can be voluntary or involuntary (Dakin et al., 2009). For voluntary averaging, only a small number of elements is presented (e.g., four: Allard \& Cavanagh, 2012; or six: Dakin et al., 2009), each element is perceptually distinct (e.g., see top row in Fig. 1), and the observer voluntarily decides to estimate the mean of an attribute (e.g., orientation) or not. The triggering of this averaging process therefore depends on the volition of the observer, so it is not compulsory or obligatory. For involuntary averaging, however, computing the mean is compulsory or obligatorythat is, beyond the volition of the observer (Dakin et al., 2009; Fischer \& Whitney, 2011; Parkes et al., 2001). Involuntary averaging typically occurs when a large amount of elements are presented in the periphery (e.g., see bottom row in Fig. 1) and is particularly relevant for scene perception as it provides a gist of the ensemble statistics and has been studied for many attributes such as orientation (e.g., Alvarez \& Oliva, 2009), size (Im \& Halberda, 2013), motion (Mareschal et al., 2008), and color (Bronfman et al., 2014).

For voluntary averaging (e.g., see top row in Fig. 1), Allard and Cavanagh (2012) concluded that observers averaged the physical (i.e., external) variance across elements, but not the internal variance resulting from imprecision estimates of individual elements by the visual system. In other words, they voluntarily and effectively averaged dissimilar elements, but not apparently identical elements. This claim was based on the psychophysical finding that performance at discriminating the mean of the a priori distribution improved with the number of samples in high variance (i.e., high jitter added to every elements), but not in low variance (i.e., identical, or nearly identical, elements and variance in sample estimates is mainly due to the sample estimates by the observers). They concluded that some voluntary averaging processing operating in high variance was not effective in low variance, which contradicts the noise-invariant processing assumption (Allard \& Cavanagh, 2011; in the current context, it could also be referred to as the "variance-invariant processing assumption") that the same processing operates in low and high variance. However, it has been argued that the result of this psychophysical study does not necessarily imply different averaging efficiencies in low and high variance, as the apparent absence of averaging efficiency in low variance could be explained by less precise estimates when there is more samples (Dakin, 2015) or by multiplicative noise (i.e., internal variance proportional to the external variance; Bocheva, Stefanov, Stefanova, \& Genova, 2015). Thus, despite the observable voluntary averaging efficiency in high variance and the absence of observable efficiency in low variance, the claim that different averaging processes operate in both conditions (i.e., the variance-invariant processing assumption underlying the equivalent noise paradigm) remains debated.

Given that voluntary averaging depends on the volition of the observer, a variance-dependent averaging process would not be surprising; why bother voluntarily averaging elements that appear identical? On the other hand, we may intuitively expect involuntary averaging processing to be independent of the variance across the elements to average. Indeed, a 


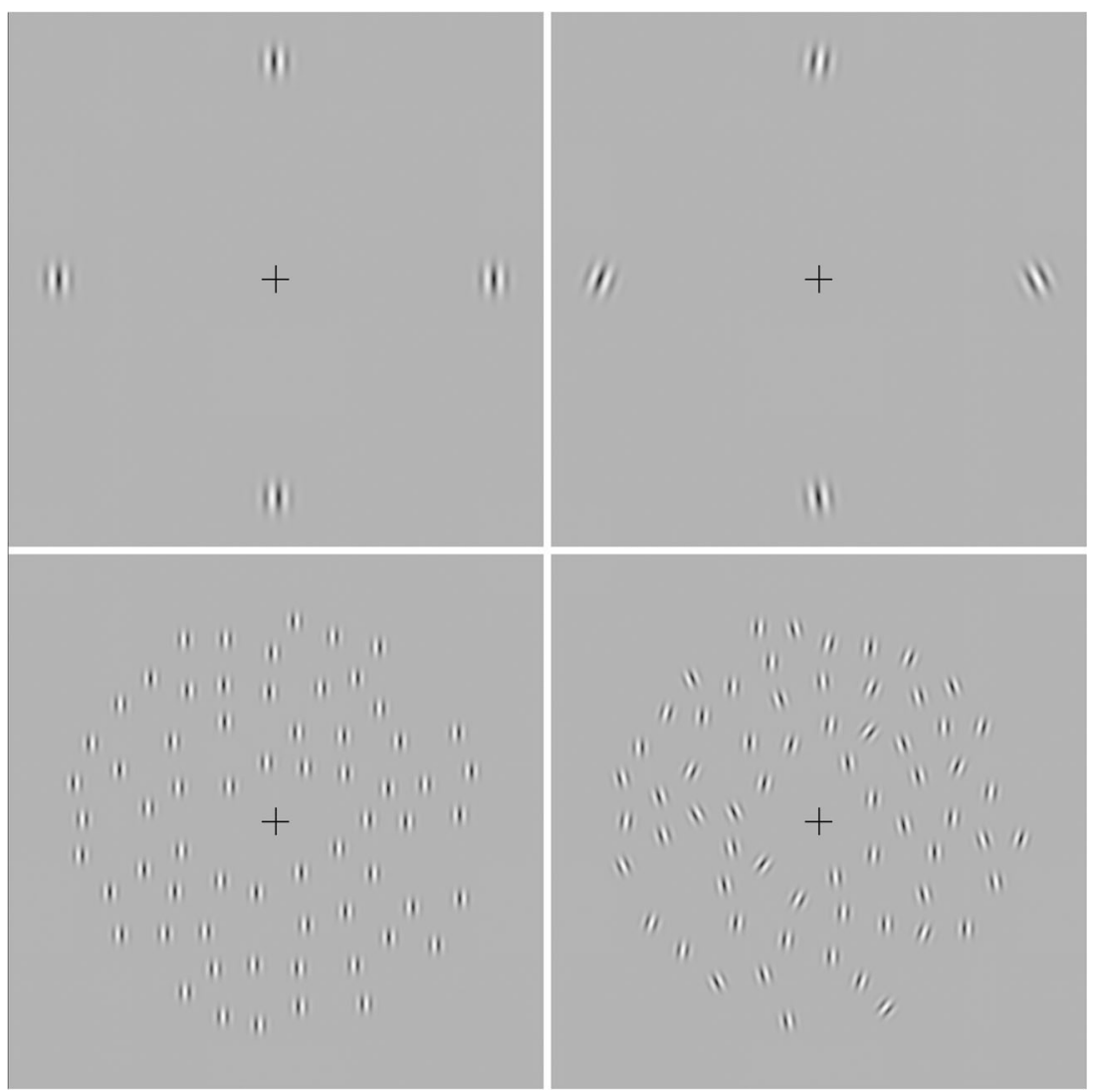

Fig. 1 Examples of stimuli for the voluntary (top) and involuntary (bottom) for low (left) or high (right) variance across samples. The task consisted in judging the mean orientation of the ensemble relative to vertical (clockwise or counterclockwise)

compulsory or obligatory averaging process would be expected to operate whether the perceived variance across elements is mainly due to the stimulus variance across elements when the variance is high or to imprecisions in the estimates of the samples when the variance is low (e.g., identical samples).

The aim of the current study was to investigate if the processing differs whether the main source of the sample imprecision is coming from the stimulus (i.e., high variance) or from the imprecision estimates of the observer (i.e., low variance). We recorded the neural activity when the main variance was coming from the stimulus and from the observer for a voluntary (i.e., four sparse elements; see top images in Fig. 1) and an involuntary (64 elements presented in the periphery; see bottom images in Fig. 1) orientation-averaging task.

\section{Method}

\section{Participants}

Seventeen young adults were included in the study (nine females; mean age $\pm S D=26.6 \pm 4.4$ years; age range: 21-38 years), but three subjects were excluded for inscanner motion (movements $>3 \mathrm{~mm}$ across trials). The participants were part of the French cohort population
SilverSight ( 350 subjects), established and followed-up ever since 2015, at Vision Institute-Quinze-Vingts National Ophthalmology Hospital, Paris, France (Lagrené et al., 2019). All participants were right-handed, had normal or corrected-to-normal vision, and they had no history of neurological or psychiatric disorders, or ocular disorders, or sensorimotor dysfunctions. All participants gave their informed written consent before participating in the study in accordance with the tenets of the Declaration of Helsinki, and they were approved by the Ethical Committee CPP Ile de France V (ID_RCB 2015-A01094-45, CPP N: 16122).

\section{Apparatus}

Stimuli were displayed using nordicAktiva software (https:// www.nordicneurolab.com/) on an MRI-compatible liquid crystal display monitor (NordicNeuroLab, Bergen, Norway) positioned at the head of the scanner bore. Participants viewed the screen (size: $69.84 \mathrm{~cm} \mathrm{[H]} \times 39.26 \mathrm{~cm}$ [V]; pixels: $1,920 \times$ 1,080; refresh rate: $120 \mathrm{~Hz}$, mean luminance intensity: $203 \mathrm{~cd} /$ $\mathrm{m}^{2}$ ) at a distance of $115 \mathrm{~cm}$ via a mirror fixed above the head coil. The visible part of the screen subtended approximately $34 \times 19$ degrees of visual angle (dva). 


\section{Stimuli and procedure}

Observers were asked to report the mean orientation of an ensemble of Gabors relative to vertical and respond as soon as possible, while answering as accurately as possible. For voluntary averaging, the stimuli were similar to the ones used in a previous study on voluntary averaging (Allard \& Cavanagh, 2012): four Gabors displayed 8 dva to the left, right, above, and below fixation (top row in Fig. 1) so that each element was perceptually distinct (Dakin et al., 2009). The spatial frequency of the Gabors was $2 \mathrm{cpd}$; their spatial envelope was a Gaussian with a standard deviation of 0.33 $\mathrm{dva}$; their contrast was maximized and their phases were randomized.

For involuntary averaging, 64 Gabors were presented in the periphery, in which case computing their mean orientation is expected to be compulsory or obligatory - that is, beyond the volition of the observer (Dakin et al., 2009; Fischer \& Whitney, 2011; Parkes et al., 2001). The Gabors were randomly positioned between 2 and 8 dva of eccentricity, with the constraint that center-to-center distance between Gabors had to be at least $1.5 \mathrm{dva}$ (see bottom row in Fig. 1). In order to increase the gap between Gabors, their Gaussian spatial envelope was smaller ( $S D$ of $0.17 \mathrm{dva}$ ) and their spatial frequency was 3 cpd.; their contrast was maximized and their phases were randomized.

In the low variance condition, the Gabors were all vertically orientated. In the high variance condition, the orientations of the Gabors were selected from a Gaussian distribution centered vertically, with a standard deviation of the distribution of 16 degrees. The stimuli were presented for $200 \mathrm{~ms}$, which was too brief for the observer to saccade to the target (Hallett, 1986).

We used a block-design paradigm with four different conditions: low-variance with four elements (LV-4; top-left in Fig. 1), low-variance with 64 elements (LV-64; bottom-left in Fig. 1), high-variance with four elements (HV-4; top-right in Fig. 1), and high-variance with 64 elements (HV-64; bottom-right in Fig. 1). A total of 120 trials were performed per condition for a total of 480 trials. These trials were separated among six functional runs presented in a random order: three runs for voluntary averaging (LV-4 and HV-4) and three runs for involuntary averaging (LV-64 and HV-64). Each functional scan lasted 5 minutes and was composed of 16 five-trial blocks, alternating between LV and HV, with the first block randomly selected (LV or HV). Each fivetrial block lasted 15 seconds. The 16 blocks were interspersed with four 15 -second blocks with a fixation cross at the center of the screen (fixation condition) displayed against a gray background.

Each stimulus was displayed for $200 \mathrm{~ms}$ followed by a fixation cross in the center of the screen displayed against a gray background. The interval between the onset of two successive stimuli was $2.8 \mathrm{~s}$. Participants had to give a categorical answer after each image by pressing the corresponding handheld grips response device (NordicNeurolab) to indicate whether the mean orientation of the Gabors was tilted clockwise or counterclockwise from vertical. They were instructed to fixate on the center of the screen (fixation cross) during the entire experiment and to respond as accurately and as quickly as possible. Response accuracy and reaction times were recorded.

A practice session in a psychophysical laboratory was conducted a few days before the experiment for participants to be familiarized with the psychophysical task and the four types of stimuli prior to the data acquisition.

\section{fMRI acquisition}

Images were acquired using a 3T Siemens Magnetom Skyra whole-body MRI system (Siemens Medical Solutions, Erlangen, Germany), with a 64-channel head coil at the Quinze-Vingts National Ophthalmology Hospital in Paris, France. Task-based fMRI and an anatomical image were acquired for all participants. The anatomical volume consisted of a T1-weighted, high-resolution, three-dimensional MPRAGE sequence (TR/TE/IT/flip angle $=2,300 \mathrm{~ms} / 2.9 \mathrm{~ms} / 900 \mathrm{~ms} /$ $9^{\circ}$; matrix size $=256 \times 240 \times 176$; voxel size $=1 \times 1 \times 1.2$ $\mathrm{mm})$. For functional scan, 304 volumes of 64 slices were acquired using a T2*-weighted SMS-EPI sequence (TR/TE/ flip angle $=1,000 \mathrm{~ms} / 30 \mathrm{~ms} / 90^{\circ}$; matrix size $=100 \times 100$; $\mathrm{SMS}=2 ;$ GRAPPA $=2$; voxel size $=2.5 \times 2.5 \times 2.4 \mathrm{~mm}$ )

\section{Data analysis}

Data analysis was performed using SPM12 release 7487 (Wellcome Department of Imaging Neuroscience, London, UK; www.fil.ion.ucl.ac.uk/spm) implemented in MATLAB 2018a (The MathWorks, Inc., Natick, MA, USA).

For each participant, the first four functional volumes of each runs were discarded to allow for equilibration effects. The remaining volumes were realigned to correct for head movements to the mean functional images using a rigid body transformation. The T1-weighted anatomical volume was then realigned (affine transformation) to match the mean functional image of each participant, and was then normalized (nonrigid, nonlinear transformation) into the MNI space. A fourthdegree B-spline interpolation was applied. The anatomical normalization parameters were subsequently used for the normalization of functional volumes. Finally, each functional scan was smoothed by an $8 \mathrm{~mm}$ FWHM (Full Width at Half Maximum) Gaussian kernel. Slice-timing correction was not applied in line with the recommendations of the Human Connectome Project functional preprocessing pipeline for multi-slice sequences (Glasser et al., 2013). 
Statistical analysis was performed using the general linear model (Friston et al., 1994) for block designs at single participant level. For each participant, five conditions of interest (LV-4, LV-64, HV-4, HV-64, and fixation) were modelled as five regressors, constructed as boxcar functions and convolved with a canonical hemodynamic response function. Reaction-time for each trial and movement parameters derived from realignment corrections (three translations and three rotations) were also considered in the model as an additional factor of no interest to account for related variance. Time series for each voxel were high-pass-filtered $(1 / 128 \mathrm{~Hz}$ cutoff) to remove low-frequency noise and signal drift. On an individual level (first-level analysis), we identified the brain regions involved in the processing of each level of variance content relative to the fixation ([LV-ALL > Fixation] and [HV-ALL > Fixation]) irrespective to the number of elements. Then, we identified the cerebral regions involved in the processing of low-variance stimuli related to the high-variance stimuli, as well as the inverse fMRI contrast ([LV-ALL > HV-ALL] and [HVALL > LV-ALL]. We also, tested the effect of number of elements ([ALL-4 > ALL-64] and [ALL-64 > ALL$4]$ ), irrespective to the variance content, and we identified brain regions involved in the processing of variance related to the number of elements ([LV-4 > HV-4], [HV-4 > LV-4], [LV-64 > HV-64], and [HV-64> LV-64]. Finally, we tested the effect of level of variance for both number of elements conditions relative to the fixation (([LV-4 > Fixation], [HV-4 > Fixation], [LV-64 > Fixation], and [HV-64 > Fixation]).

To allow population inference, we performed a second level-random analysis using a one-sample $t$ test. Areas of activation were considered significant if they exceed a significant threshold fixed at $p<.05$ FWEcorrected for multiple comparisons at cluster level, with a minimum cluster extend $\mathrm{k}=20$.

\section{Results}

\section{Behavior}

The percentage of trials on which participants did not give an answer within 2.8 seconds was lower than $1 \%$ for each of the four conditions $(\mathrm{LV}-4=0.6 \%$; HV-4 $=0.8 \%$; LV- $64=0.5 \%$, and HV-64 $=0.5 \%)$. These results confirm that participants followed the instructions to respond as fast as possible. Noresponse trials were removed from behavioral analyses.

Figure 2 shows the number of participants who answered clockwise for each stimulus as a function of the mean of the ensemble for conditions in high variance. With four Gabors (left graph in Fig. 2), the mean orientation of the stimulus varied considerably across stimuli, and the participants' answers were highly correlated with the stimulus' mean orientation as confirmed by a linear regression analysis $(b=0.38)$, $t(11)=16.7, p<.001$. Indeed, when the mean orientation was highly tilted clockwise, almost all of participants answered "clockwise", and when the mean orientation was highly tilted counterclockwise, almost all of them answered "counterclockwise" (i.e., just a few answered "clockwise"). As a result, the participants' answers were stimulus driven, not random (see Fig. 3). With 64 Gabors (right graph in Fig. 2), the stimulus' mean orientation was much closer to vertical due to the greater number of elements. Although the participants' answers were less correlated with the actual stimulus mean (see Fig. 3), the participants' answers also significantly depended on the mean orientation $(b=0.40), t(11)=2.93, p<.01$. These results confirm that participants effectively performed the task of reporting the mean orientation relative to vertical (i.e., they did not answer randomly).

The distributions for the reaction times and the median reaction times (see Fig. 4) were highly similar for the four conditions. The reaction times for the four conditions were

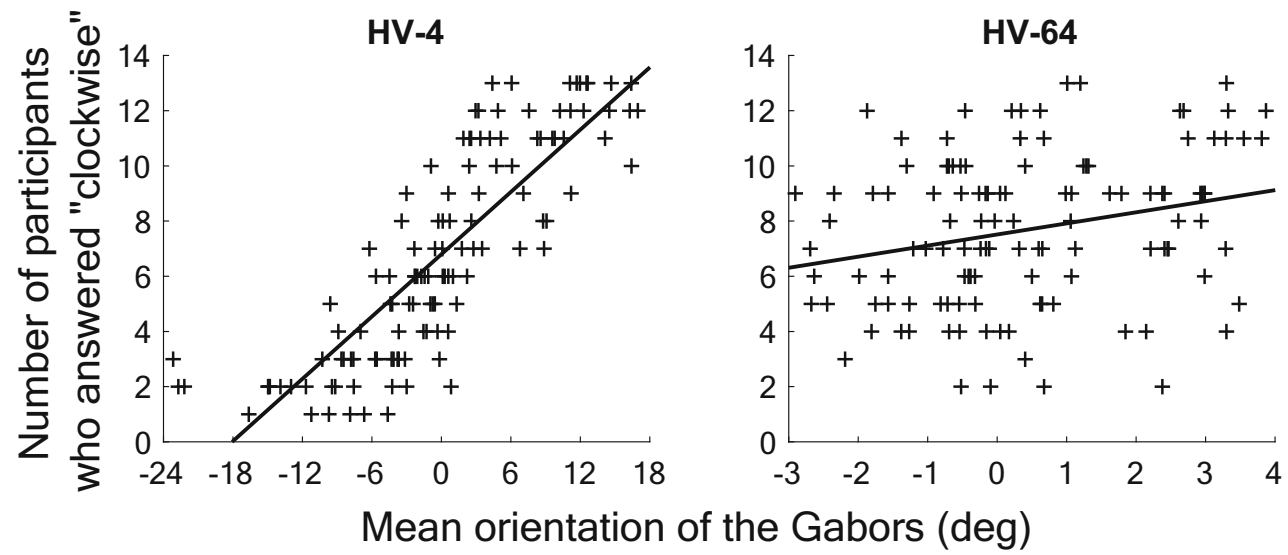

Fig. 2 Number of participants who answered "clockwise" as a function of the mean of the ensemble for each of the 120 stimuli in high variance with 4 Gabors (HV-4, left) and with 64 Gabors (HV-64, right). The solid lines represent the linear regressions 


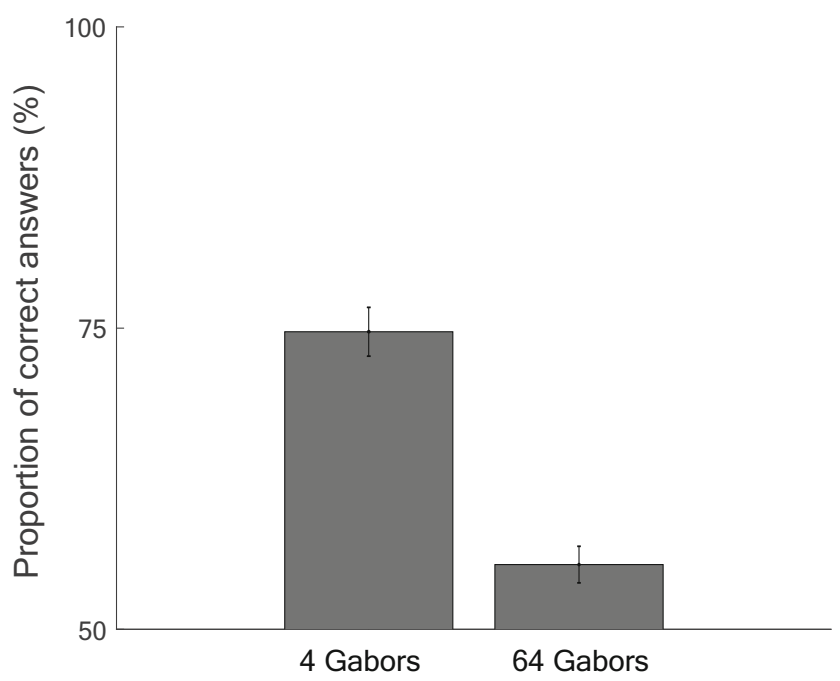

Fig. 3 Proportion of correct answers for both tasks in high variance. Error bars represent standard error from the mean

analyzed with an inverse Gaussian generalized linear-mixed model with an identity link, and no significant effect was found for the two fixed factors, which were the variance (estimate $=12.15, t=1.25, p=.21)$ and the number of elements (estimate $=-0.26, t=-1.58, p=.11$ ). The fact that similar reaction times were observed in low and high variance is compatible with the hypothesis that the processing was independent of the variance across elements (i.e., the varianceinvariant processing assumption). Consequently, from the behavioral data alone, there was no evidence of different processing involved in low and high variance (but see fMRI results below).

\section{fMRI}

Effect of variance content Results for the effect of variance content relative to the fixation, irrespective to the number of elements are shown in Table 1 and Fig. 5. Results for direct comparisons of variance conditions are presented in Table 2 and Fig. 6.

We began by contrasting the processing of Gabor orientation in low-variance to fixation ([(LV-ALL > Fix ] contrast), and observed recruitment of the superior parietal and the superior frontal gyri in the left hemisphere. Similarly, the processing of Gabor orientation in high-variance condition ([HVALL > Fix]) showed activation of the superior parts of the parietal and frontal gyri in the left hemisphere, and supplementary involvement of the middle frontal gyrus bilaterally. Direct comparisons of variance contents showed no significant results for low-variance to high-variance ([LV-ALL > HV-ALL]). The opposite contrast ([HV-ALL > LV-ALL]) elicited activations in the cerebellum, the inferior occipital gyrus and the middle frontal gyrus in the left hemisphere.
Critically, the direct comparison of variance conditions related to the number of elements showed significant results only for the contrast [HV-64 > LV-64] involving the recruitment of the left middle frontal gyrus and the superior frontal gyrus bilaterally (mainly in the right hemisphere; see Table 3 and Fig. 7).

Effect of number of elements Direct comparisons of the number of elements irrespective to the variance contents for Gabor orientations ([ALL-4 > ALL-64] and [ALL-64 > ALL-4] showed no significant results. Results for the effect of the number of elements related to the variance contents compared with the fixation condition are shown in the Supplementary Information section (Table S1 and Fig. S1).

Results for the processing of variance contents related to the number of elements relative to the fixation showed activation for high variance only for four Gabors and for both lowvariances and high-variances conditions for 64 Gabors. Precisely, these three contrasts ([HV-4 > Fix], [LV-64 > Fix], and [HV-64 > Fixation] involved common cerebral structures with the left superior parietal gyrus, the left middle, and superior frontal gyri. In addition, the contrast [HV-4 > Fix] elicited specific activation of the middle and superior frontal gyri in the right hemisphere whereas, the contrast [LV-64 > Fix] elicited activation located in the left supramarginal gyrus. Finally, the contrast [HV-64 > Fix] showed significant results also on the left supramarginal gyrus and the right middle occipital gyrus.

\section{Discussion}

The reaction times around 1 second are consistent with a previous study (Bocheva et al., 2015) in which participants were also asked to respond as fast as possible (while being accurate). The fact that the reaction times in the current study did not vary with the variance across samples (i.e., between LV and $\mathrm{HV}$ ) is consistent with the variance-invariant processing assumption: in low variance, imprecisions in sample estimates introduces variance across samples and the observer averages these estimates as they do in high variance. These results diverge from the ones of Bocheva and colleagues, who found reaction times longer with higher variance stimuli, but can be explained by the task difficulty. In Bocheva's study, the same signal strengths (i.e., mean of the a priori distribution) were used at all variances, but mean orientation discrimination thresholds were higher with higher variance, so the task was, on average, more difficult at higher variance (lower signal strength relative to the participants' thresholds). In the current study, all conditions were below thresholds as the mean of the a priori distribution was zero (note that according to the equivalent noise paradigm, the correctness must be defined relative to the mean of the a priori distribution, not the actual mean of 

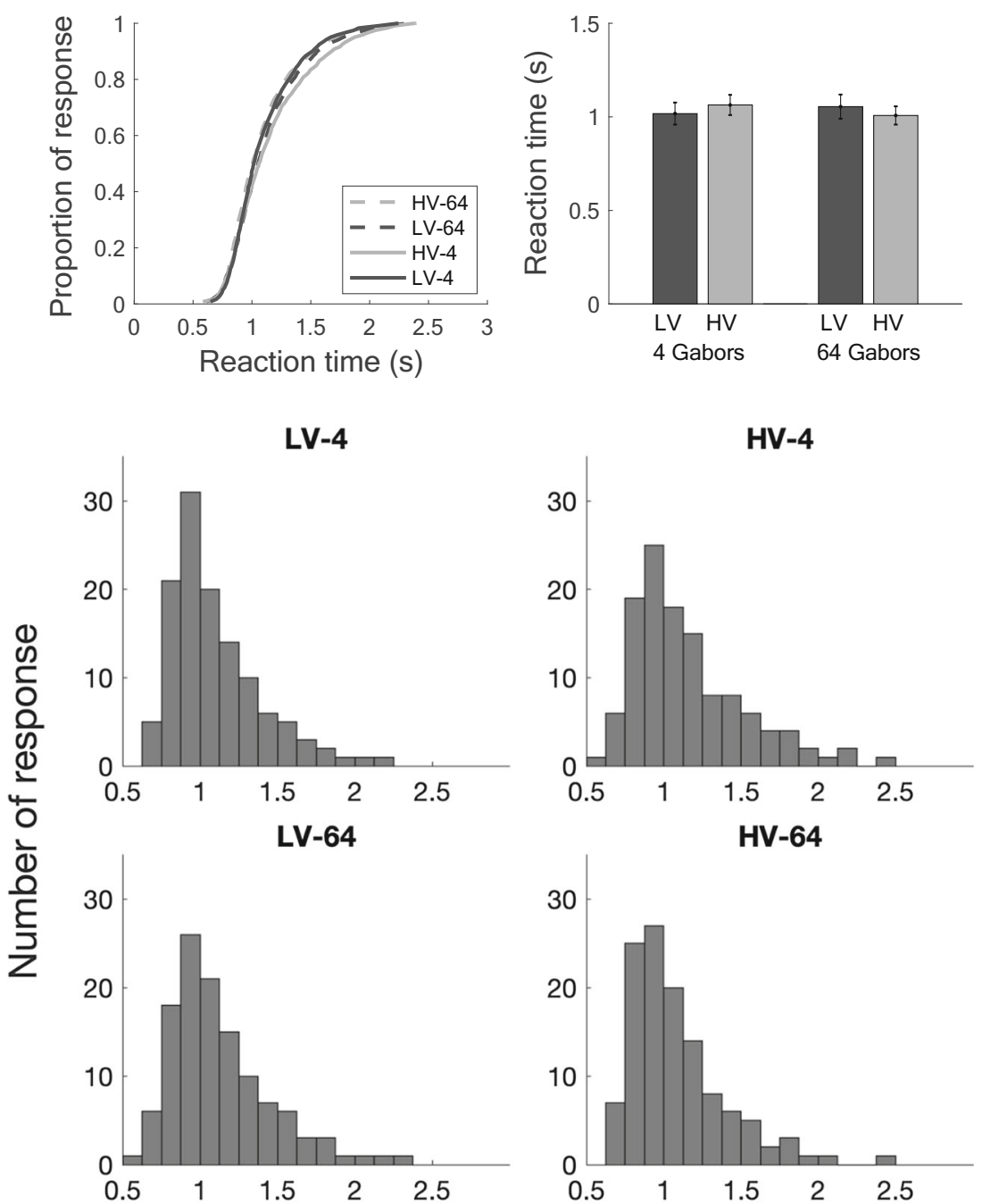

Reaction time (s)

Fig. 4 Proportion of response given as a function of the reaction time for the four conditions with all the participants (top left). Mean median reaction times for the four conditions averaged across observers (top

the stimulus). Thus, without a signal strength greater relative to threshold, we did not observe in the behavioral data a violation of the variance-invariant processing assumption - that is, that the same averaging processing operates independently of the source of the main variance (imprecisions in sample estimates or the stimulus). Conversely, the results regarding the response time suggests that the different reaction times observed at different levels of variance in a previous study was due to a confounding factor (i.e., task difficulty), not variance level per se.

The fMRI data showed that low-variance and highvariance elicited neural activations within common brain regions including the superior parietal and frontal gyri in the left hemisphere. These cortical regions are known to be involved in calculation tasks (Fehr, Code, \& Herrmann, 2007; Rickard et al., 2000; Rosenberg-Lee, Chang, Young, Wu, \& Menon, 2011) and particularly in the left hemisphere (Arsalidou \& right). $\mathrm{LV}=$ low variance condition and $\mathrm{HV}=$ high variance condition. Error bars represent standard error from the mean. Histograms of the reaction times for the four conditions (bottom)

Taylor, 2011). Critically, our parietal cluster in the left hemisphere ( $x=-35, y=-42, z=53)$ appeared to be very close to the brain coordinate located in the horizontal segment of the intraparietal sulcus $(x=-37, y=-48, z=49)$ reported by Andres, Pelgrims, Michaux, Olivier, and Pesenti (2011), and mainly involved on number magnitude processing (Dehaene, Piazza, Pinel, \& Cohen, 2003) or division operations (Andres et al., 2011). The similar activation of brain areas typically related to arithmetic calculation and orientation averaging begs the speculation of a calculation that is not specific to arithmetic. Further investigations are required to investigate this speculative link.

Although no behavioral differences were observed between averaging in low and high variance, and similar brain areas were activated, some differences in neural activity amplitude were observed. The right superior frontal and left middle frontal gyri were significantly more activated when the 
Table 1 Cerebral regions exhibiting an effect of the level of variance (low-variance stimuli: LV-ALL and high-variance stimuli: HV-ALL) related to fixation (Fix)

\begin{tabular}{|c|c|c|c|c|c|c|c|c|}
\hline & & $\mathrm{H}$ & BA & $\mathrm{k}$ & $x$ & $y$ & $z$ & $t$ \\
\hline \multicolumn{9}{|l|}{ fMRI contrasts } \\
\hline \multirow[t]{3}{*}[\mathbf{LV}-\mathbf{ALL}>\text{Fix}]{} & Superior parietal gyrus & $\mathrm{L}$ & 7 & 94 & -35 & -42 & 53 & 9.16 \\
\hline & [Postcentral gyrus] & & 1 & & -40 & -35 & 48 & 7.10 \\
\hline & Superior frontal gyrus & $\mathrm{L}$ & 6 & 49 & -17 & 1 & 50 & 6.81 \\
\hline \multirow[t]{7}{*}[\mathrm{HV}-\mathrm{ALL}>\mathrm{Fix}]{} & Superior parietal gyrus & $\mathrm{L}$ & 7 & 141 & -35 & -42 & 53 & 11.22 \\
\hline & & & & & -40 & -35 & 48 & 8.40 \\
\hline & & & & & -42 & -40 & 58 & 6.65 \\
\hline & Superior frontal gyrus & $\mathrm{L}$ & 6 & 191 & -17 & 3 & 53 & 7.54 \\
\hline & [Middle frontal gyrus] & & & & -30 & 1 & 60 & 7.54 \\
\hline & [Middle frontal gyrus] & & & & -25 & -5 & 50 & 6.85 \\
\hline & Middle frontal gyrus & $\mathrm{R}$ & 6 & 36 & 26 & -2 & 53 & 6.40 \\
\hline
\end{tabular}

Note. The statistical threshold for cluster was defined as $p<.05$ FWE corrected for multiple comparisons with an extent voxel threshold defined as 20 voxels. Only regions revealing significant differences between conditions were included. For each cluster, the region showing the maximum $t$ value was listed first, followed by the other regions in the cluster [in square brackets]. Montreal Neurological Institute (MNI) coordinates $(x, y, z)$ of the peak and number of voxels $(\mathrm{k})$ of clusters are also shown. $\mathrm{H}=$ hemisphere; $\mathrm{R}=$ right hemisphere; $\mathrm{L}=$ left hemisphere; $\mathrm{BA}=\mathrm{Brodmann}$ area

variance in the orientation of the Gabors was high than when it was low. The direct comparison between high and low variance conditions also elicited an unexpected cerebral activity overlapping the cerebellum (Crus I) and the inferior occipital gyrus in the left hemisphere. Andres et al. (2011) reported a similar cluster for arithmetic operation (multiplication and subtraction), but in the right hemisphere. Conversely, no cortical area showed significantly more neural activation in the low variance conditions compared with the high variance conditions. Thus, more neural activity was observed when estimating the mean orientation of a high-variance ensemble compared with estimating the mean orientation of a low-variance ensemble.
The additional activation that occurred in high variance relative to low variance appears to be mainly driven by the 64-Gabor condition and not by the four-Gabor condition. These results are surprising to us. As mentioned in the introduction, based on a previous psychophysical study (Allard \& Cavanagh, 2011), we could expect voluntary averaging (i.e., four Gabor) to occur when the elements are dissimilar (i.e., high variance), but not when they appear identical (i.e., low variance), because observers may not bother averaging samples that appear identical. Interestingly, however, additional activation in the right superior frontal and left middle frontal gyri was observed in high variance relative to low variance for involuntary averaging (i.e., 64-Gabor condition). This result
[LV-ALL > Fix]

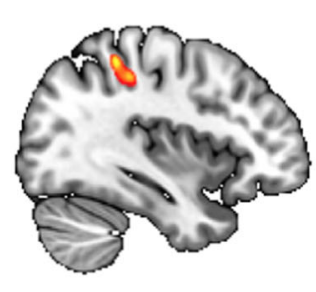

$x=-37$

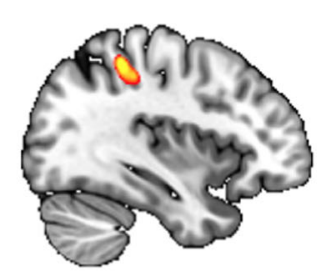

$x=-37$

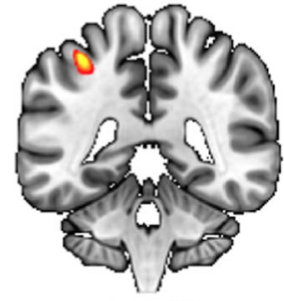

$y=-42$

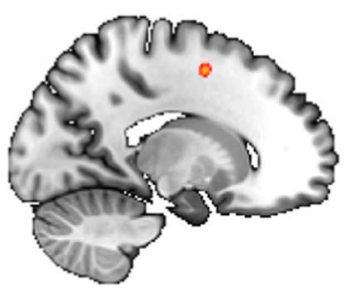

$x=-17$

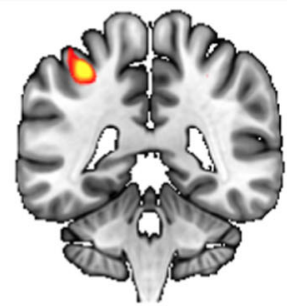

$y=-42$

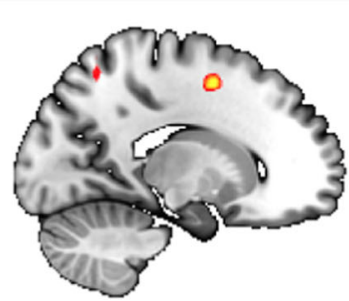

$x=-17$

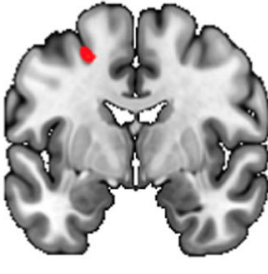

$y=-4$

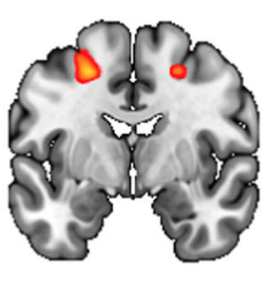

$y=-4$
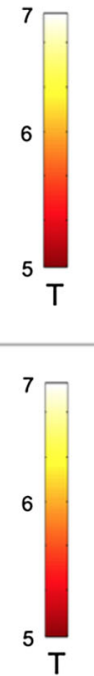

Fig. 5 Cerebral regions whose activity was elicited by the fMRI contrasts [LV-ALL $>$ Fix] and [HV-ALL $>$ Fix] projected onto $2 \mathrm{D}$ slices $(p<.05$ FWEcorrected at cluster level, $\mathrm{k}=20 ; \mathrm{LV}=$ low-variance; HV = high-variance; Fix = fixation) 
Table 2 Cerebral regions exhibiting an effect for direct comparison of variance contents between low-variance (LV-ALL) and high-variance (HVALL) stimuli

\begin{tabular}{|c|c|c|c|c|c|c|c|c|}
\hline & & $\mathrm{H}$ & BA & $\mathrm{k}$ & $x$ & $y$ & $z$ & $t$ \\
\hline \multicolumn{9}{|l|}{ fMRI contrasts } \\
\hline$[\mathbf{L V}-\mathbf{A L L}>\mathbf{H V}$-ALL] & No significant activation & & & & & & & \\
\hline \multirow[t]{4}{*}{ HV-ALL > LV-ALL] } & Cerebellum & $\mathrm{L}$ & - & 59 & -30 & -75 & -30 & 6.22 \\
\hline & [Inferior occipital gyrus] & & 19 & & -40 & -77 & -18 & 6.07 \\
\hline & Middle frontal gyrus & $\mathrm{L}$ & 6 & 37 & -35 & 6 & 38 & 6.33 \\
\hline & [Middle frontal gyrus] & & & & -30 & -16 & 38 & 5.79 \\
\hline
\end{tabular}

Note. The statistical threshold for cluster was defined as $p<.05$ FWE corrected for multiple comparisons with an extent voxel threshold defined as 20 voxels. Only regions revealing significant differences between conditions were included. For each cluster, the region showing the maximum $t$ value was listed first, followed by the other regions in the cluster [in square brackets]. Montreal Neurological Institute (MNI) coordinates $(x, y, z)$ of the peak and number of voxels $(\mathrm{k})$ of clusters are also shown. $H$ hemisphere, $R$ right hemisphere, $L$ left hemisphere, $B A$ Brodmann area

was less expected, but has more impact given that most studies on ensemble statistics focus on large ensembles (e.g., 64) rather than small ensembles as in voluntary averaging.

The current finding showing additional activation in the left middle frontal gyrus with 64 samples in high variance relative to low variance is consistent with the hypothesis that some of this additional processing is related to averaging calculation. Indeed, the middle frontal gyrus from both hemispheres has been found to be involved in mental calculations involving working memory, especially for complex tasks (Arsalidou \& Taylor, 2011). Similarly, bilateral involvement of the frontal regions in both hemispheres for the fMRI contrast [HV-64 > LV-64] suggested that supplementary processing was needed during the high variance condition (Fehr et al., 2007). Consequently, the current findings with 64 elements to average, are consistent with the hypothesis that more processing relevant to the averaging calculation occurs when the main source of variance was due to physical variance across elements (i.e., high variance) than when the source of variance was due to imprecision estimates by the visual system (i.e., low variance). Further studies are required to test this hypothesis.

It is also possible that the different activation is caused by different efforts by the observer due to the apparent task difficulty. Some observers have reported that the task seemed more difficult when all the orientations were close to vertical, and others actually reported that the task was more difficult when the orientations of the Gabors were dissimilar because it was more difficult to average. Thus, it is possible that observers reduced their effort in low variance because they gave up more in low variance (task appeared too difficult) or because they put more efforts in high variance as the tasked appeared more difficult. If the observers modulated their effort based on the apparent task difficulty and the effort level affects the averaging efficiency, then this would obviously violate the variance-invariant processing assumption, as different effective averaging efficiencies would occur in low and high variance. In other words, using the equivalent noise paradigm for

\section{[HV-ALL > LV-ALL]}
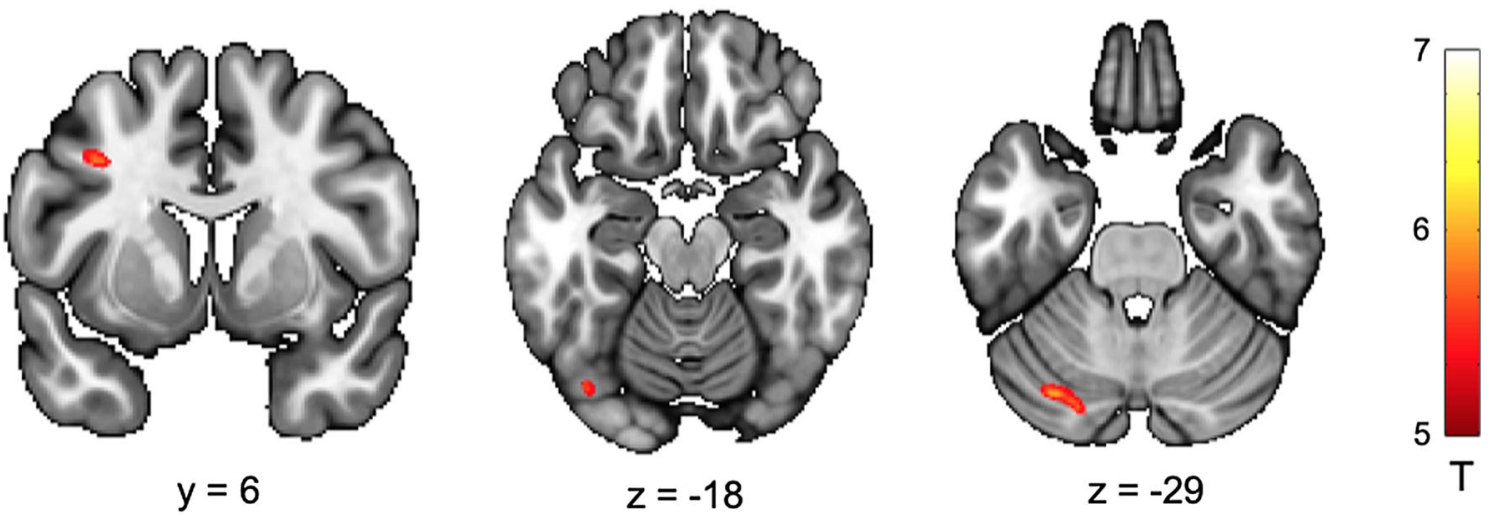

Fig. 6 Cerebral regions whose activity was elicited by the fMRI contrast [HV-ALL $>$ LV-ALL] projected onto 2D slices $(p<.05$ FWE-corrected at cluster level, $\mathrm{k}=20 ; \mathrm{LV}=$ low variance, $\mathrm{HV}=$ high variance) 
Table 3 Cerebral regions exhibiting an effect for direct comparisons between variances conditions related to the number of elements (lowvariance with four elements: LV-4, high-variance with four elements:
HV-4, low-variance with 64 elements: LV-64 and high-variance with 64 elements: HV-64) related to fixation (Fix)

\begin{tabular}{|c|c|c|c|c|c|c|c|c|}
\hline & & $\mathrm{H}$ & $\mathrm{BA}$ & $\mathrm{k}$ & $x$ & $y$ & $z$ & $t$ \\
\hline \multicolumn{9}{|l|}{ fMRI contrasts } \\
\hline [LV-4 > HV-4] & No significant activation & & & & & & & \\
\hline$[\mathrm{HV}-4$ > LV-4] & No significant activation & & & & & & & \\
\hline [LV-64 > HV-64] & No significant activation & & & & & & & \\
\hline \multirow[t]{2}{*}{ [HV-64 > LV-64] } & Middle frontal gyrus & $\mathrm{L}$ & 8 & 93 & -37 & 11 & 40 & 7.43 \\
\hline & Superior frontal gyrus & $\mathrm{R}$ & 8 & 33 & 6 & 31 & 50 & 6.89 \\
\hline$[\mathrm{LV}-4>\mathrm{HV}-64]$ & No significant activation & & & & & & & \\
\hline$[\mathrm{HV}-64>\mathrm{LV}-4]$ & No significant activation & & & & & & & \\
\hline$[\mathrm{LV}-64>\mathrm{HV}-4]$ & No significant activation & & & & & & & \\
\hline$[\mathrm{HV}-4$ > LV-64] & No significant activation & & & & & & & \\
\hline
\end{tabular}

Note. The statistical threshold for cluster was defined as $p<.05$ FWE corrected for multiple comparisons with an extent voxel threshold defined as 20 voxels. Only regions revealing significant differences between conditions were included. Montreal Neurological Institute (MNI) coordinates $(x, y$, $z$ ) of the peak and number of voxels $(\mathrm{k})$ of clusters are also shown. $\mathrm{H}=$ hemisphere; $\mathrm{R}=$ right hemisphere; $\mathrm{L}=$ left hemisphere; $\mathrm{BA}=\mathrm{Brodmann}$ area

an averaging task requires the assumption that the observer's averaging efforts are not modulated by the apparent task difficulty that may differ between low and high variance.

Note that we are not assuming that the task difficulty is the same in low and high variance; it is the typical equivalent noise paradigm that relies on the assumption that the task difficulty is the same in these two conditions. In high variance, the samples are all vertically oriented (mean of the a priori distribution) and some orientation noise jitter (variance of the a priori distribution) is added to each sample. In absence of stimulus variance, the samples are all vertically oriented, and some orientation noise jitter (due to imprecision in sample estimates by the visual system; i.e., internal noise) is added to each sample. Thus, the effective stimulus (i.e., samples after the internal noise is added) is supposed to be equivalent according to the assumption underlying the equivalent noise paradigm (see the noise-invariant processing assumption: Allard \& Cavanagh, 2011, 2012; and the contrast-invariant calculation assumption: Pelli, 1990): Samples are vertically oriented and some orientation noise jitter is added. Consequently, according to the equivalent noise paradigm, the task difficulty is assumed not to differ between low and high variance when the a priori distribution is centered on vertical. If the task difficulty differed between low and high variance conditions, then this would undermine the use of the equivalent noise paradigm. In sum, the equivalent noise paradigm requires the assumption that the task difficulty in low and high variance did not differ and that observers put the same effort in low and high variance.

Nevertheless, it is possible that the effective averaging efficiency was the same in low and high variance, despite the fact that there was more neural activity in high variance. The additional neural activity in brains areas related to arithmetic calculation may not be related to the averaging process. For instance, orientation uniformity in low variance could lead to greater suppression/inhibition in early visual areas and thus

[HV-64 > LV-64]
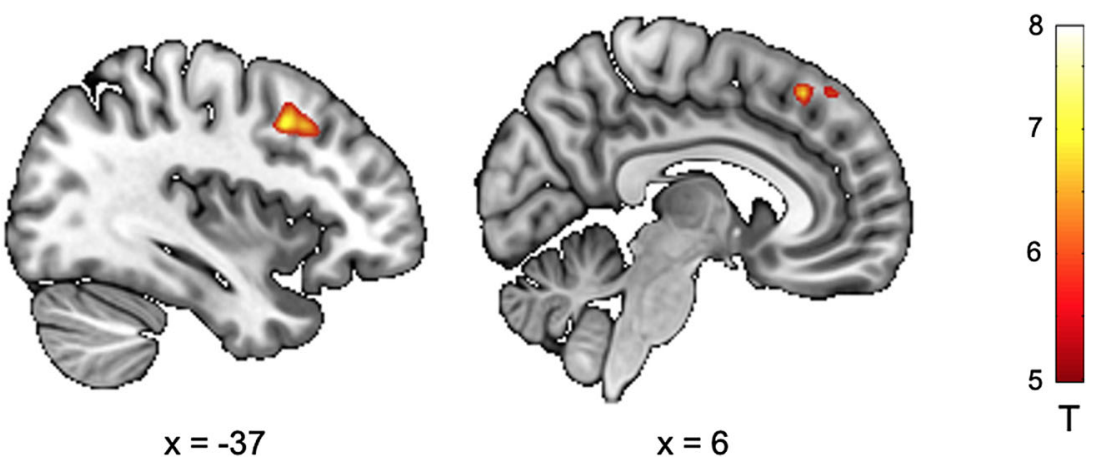

Fig. 7 Cerebral regions whose activity was elicited by the fMRI contrast [HV-64 $>$ LV-64] projected onto 2D slices $(p<.05$ FWE-corrected at cluster level, $\mathrm{k}=20 ; \mathrm{LV}=$ low-variance, $\mathrm{HV}$ = high-variance) 
reduced activity is fed forward to later visual areas, or higher variance could lead to richer accidental contour structure activating later form-selective areas more effectively, or higher variance may produce more attentionally engaging accidental stimuli, solicitating more task-unrelated attention. If the additional neural activity in high noise is not caused by the averaging calculation per se and it does not affect indirectly the averaging process (e.g., through lateral inhibition between processes or competing attentional resources), then it would be justified to assume that the same effective averaging efficiency operates in low and high variance. On the other hand, if the additional neural activity reflects more processing related to the involuntary averaging computation, or if it reflects different efforts due to apparent task difficulty, or if it causes some other process to indirectly affect the averaging computation, then it would undermine the assumption that the effective averaging efficiency is the same in low and high variance. Further studies are required to determine whether this additional neural activity affects directly or indirectly the effective averaging efficiency.

Supplementary Information The online version contains supplementary material available at https://doi.org/10.3758/s13414-020-02223-8.

Acknowledgements This research was supported by the Chair SILVERSIGHT ANR-18-CHIN-0002 and by the IHU FOReSIGHT ANR-18-IAHU-01. The authors thank the participants of this study for their valuable contributions. We thank the CHNO des Quinze-Vingts for enabling us to perform the MRI acquisitions.

\section{References}

Allard, R., \& Cavanagh, P. (2011). Crowding in a detection task: external noise triggers change in processing strategy. Vision Research, 51(4), 408-416.

Allard, R., \& Cavanagh, P. (2012). Different processing strategies underlie voluntary averaging in low and high noise. Journal of Vision, 12(11). https://doi.org/10.1167/12.11.6

Allard, R., \& Faubert, J. (2013). Zero-dimensional noise is not suitable for characterizing processing properties of detection mechanisms. Journal of Vision, 13(10). https://doi.org/10.1167/13.10.25

Allard, R., \& Faubert, J. (2014). To characterize contrast detection, noise should be extended, not localized. Frontiers in Psychology, 5(749). https://doi.org/10.3389/fpsyg.2014.00749

Alvarez, G. A., \& Oliva, A. (2008). The representation of simple ensemble visual features outside the focus of attention: Research article. Psychological Science, 19(4), 392-398. https://doi.org/10.1111/j. 1467-9280.2008.02098.x

Alvarez, G. A., \& Oliva, A. (2009). Spatial ensemble statistics are efficient codes that can be represented with reduced attention. Proceedings of the National Academy of Sciences of the United States of America, 106(18), 7345-7350. https://doi.org/10.1073/ pnas.0808981106

Andres, M., Pelgrims, B., Michaux, N., Olivier, E., \& Pesenti, M. (2011). Role of distinct parietal areas in arithmetic: An fMRI-guided TMS study. NeuroImage https://doi.org/10.1016/j.neuroimage.2010.11. 009
Arsalidou, M., \& Taylor, M. J. (2011). Is $2+2=4$ ? Meta-analyses of brain areas needed for numbers and calculations. Neurolmage https://doi.org/10.1016/j.neuroimage.2010.10.009

Baker, D. H., \& Meese, T. S. (2012). Zero-dimensional noise: The best mask you never saw. Journal of Vision, 12(10). https://doi.org/10. $1167 / 12.10 .20$

Baker, D. H., \& Vilidaite, G. (2014). Broadband noise masks suppress neural responses to narrowband stimuli. Frontiers in Psychology, 5(763). https://doi.org/10.3389/fpsyg.2014.00763

Baldwin, A. S., Baker, D. H., \& Hess, R. F. (2016). What do contrast threshold equivalent noise studies actually measure? Noise vs. nonlinearity in different masking paradigms. PLOS ONE, 11(3), 1-25. https://doi.org/10.1371/journal.pone.0150942

Beaudot, W. H. A., \& Mullen, K. T. (2006). Orientation discrimination in human vision: Psychophysics and modeling. Vision Research, 46(1-2), 26-46. Retrieved from http://www.sciencedirect.com/ $\mathrm{sci}$ e n c e / a r t i c 1 e/ B 6 T 0 W - 4 H P D 3 J T - 1/2/ d729e222ab5dd9a8a454ef8eb61f074b

Bocheva, N., Stefanov, S., Stefanova, M., \& Genova, B. (2015). Global orientation estimation in noisy conditions. Acta Neurobiologiae Experimentalis, 75(4), 412-433.

Bronfman, Z. Z., Brezis, N., Jacobson, H., \& Usher, M. (2014). We See more than we can report: "Cost free" color phenomenality outside focal attention. Psychological Science, 25(7), 1394-1403. https:// doi.org/10.1177/0956797614532656

Chong, S. C., \& Treisman, A. (2005). Statistical processing: Computing the average size in perceptual groups. Vision Research, 45(7), 891900. https://doi.org/10.1016/j.visres.2004.10.004

Dakin, S. C. (2001). Information limit on the spatial integration of local orientation signals. Journal of the Optical Society of America A, 18(5), 1016-1026. Retrieved from http://josaa.osa.org/abstract. cfm?URI=josaa-18-5-1016

Dakin, S. C. (2015). Seeing statistical regularities: Texture and pattern perception. In J. Wagemans (Ed.), The Oxford handbook of perceptual organization (pp. 150-167). https://doi.org/10.1093/oxfordhb/ 9780199686858.013.054

Dakin, S. C., Bex, P. J., Cass, J. R., \& Watt, R. J. (2009). Dissociable effects of attention and crowding on orientation averaging. Journal of Vision, 9(11), 1-16. https://doi.org/10.1167/9.11.28

Dakin, S. C., Mareschal, I., \& Bex, P. J. (2005). Local and global limitations on direction integration assessed using equivalent noise analysis. Vision Research, 45(24), 3027-3049. https://doi.org/10.1016/j. visres.2005.07.037

Dehaene, S., Piazza, M., Pinel, P., \& Cohen, L. (2003). Three parietal circuits for number processing. Cognitive Neuropsychology. https:// doi.org/10.1080/02643290244000239

Fehr, T., Code, C., \& Herrmann, M. (2007). Common brain regions underlying different arithmetic operations as revealed by conjunct fMRI-BOLD activation. Brain Research. https://doi.org/10.1016/j. brainres.2007.07.043

Fischer, J., \& Whitney, D. (2011). Object-level visual information gets through the bottleneck of crowding. Journal of Neurophysiology, 106(3), 1389-1398. https://doi.org/10.1152/jn.00904.2010

Friston, K. J., Holmes, A. P., Worsley, K. J., Poline, J. -P, Frith, C. D., \& Frackowiak, R. S. J. (1994). Statistical parametric maps in functional imaging: A general linear approach. Human Brain Mapping. https://doi.org/10.1002/hbm.460020402

Glasser, M. F., Sotiropoulos, S. N., Wilson, J. A., Coalson, T. S., Fischl, B., Andersson, J. L., et al. (2013). The minimal preprocessing pipelines for the human connectome project. Neuroimage 80, 105-124. https://doi.org/10.1016/j.neuroimage.2013.04.127

Hallett, P. E. (1986). Eye movements. In K. R. Boff, L. Kaufman, \& J. P. Thomas (Eds.), Handbook of perception and human performance (pp. 10-90). New York: Wiley.

Im, H. Y., \& Halberda, J. (2013). The effects of sampling and internal noise on the representation of ensemble average size. Attention, 
Perception, \& Psychophysics, 75(2), 278-286. https://doi.org/10. 3758/s13414-012-0399-4

Lagrené, K., Bécu, M., Seiple, W. H., Raphanel Bataille, M., Combariza, S., Paques, M., ... Arleo, A. (2019). Healthy and pathological visual aging in a French follow-up cohort study. Investigative Ophthalmology \& Visual Science, 60, 5915.

Mansouri, B., Allen, H. A., Hess, R. F., Dakin, S. C., \& Ehrt, O. (2004). Integration of orientation information in amblyopia. Vision Research, 44(25), 2955-2969. https://doi.org/10.1016/j.visres. 2004.06.017

Mareschal, I., Bex, P. J., \& Dakin, S. C. (2008). Local motion processing limits fine direction discrimination in the periphery. Vision Research, 48(16), 1719-1725. https://doi.org/10.1016/j.visres. 2008.05.003

Parkes, L., Lund, J., Angelucci, A., Solomon, J. A., \& Morgan, M. (2001). Compulsory averaging of crowded orientation signals in human vision. Nature Neuroscience, 4(7), 739-744. https://doi. org $/ 10.1038 / 89532$

Pelli, D. G. (1990). The quantum efficiency of vision. In C. Blakemore (Ed.), Visual coding and efficiency (pp. 3-24). Cambridge: Cambridge University Press.

Pylyshyn, Z. W., \& Storm, R. W. (1988). Tracking multiple independent targets: Evidence for a parallel tracking mechanism. Spatial Vision, 3(3), 179-197. https://doi.org/10.1163/156856888X00122

Rickard, T. C., Romero, S. G., Basso, G., Wharton, C., Flitman, S., \& Grafman, J. (2000). The calculating brain: An fMRI study. Neuropsychologia https://doi.org/10.1016/S0028-3932(99)00068-8
Rosenberg-Lee, M., Chang, T. T., Young, C. B., Wu, S., \& Menon, V. (2011). Functional dissociations between four basic arithmetic operations in the human posterior parietal cortex: A cytoarchitectonic mapping study. Neuropsychologia https://doi.org/10.1016/j. neuropsychologia.2011.04.035

Tibber, M. S., Anderson, E. J., Bobin, T., Carlin, P., Shergill, S. S., \& Dakin, S. C. (2015). Local and global limits on visual processing in schizophrenia. PLOS ONE, 10(2). https://doi.org/10.1371/journal. pone. 0117951

Treisman, A. M., \& Gelade, G. (1980). A feature-integration theory of attention. Cognitive Psychology, 12(1), 97-136.

Whitney, D., \& Yamanashi Leib, A. (2018). Ensemble Perception. Annual Review of Psychology, 69(1), 105-129. https://doi.org/10. 1146/annurev-psych-010416-044232

Open practices statement

The data sets generated during and/or analyzed during the current study are available from the corresponding author on reasonable request. The experiment was not preregistered.

Publisher's note Springer Nature remains neutral with regard to jurisdictional claims in published maps and institutional affiliations. 